\title{
Morphological and mechanical properties of carbon-nanotube-reinforced semicrystalline and amorphous polymer composites
}

\author{
M. Cadek, J. N. Coleman, ${ }^{\text {a) }}$ and V. Barron \\ Materials Ireland Polymer Research Centre, Department of Physics, Trinity College Dublin, \\ Dublin 2, Ireland \\ K. Hedicke \\ Department of Polymer Engineering, Technical University of Hamburg-Harburg, 21079 Hamburg, Germany \\ W. J. Blau \\ Materials Ireland Polymer Research Centre, Department of Physics, Trinity College Dublin, \\ Dublin 2, Ireland
}

(Received 16 September 2002; accepted 5 November 2002)

\begin{abstract}
In this work, multiwalled carbon nanotubes were investigated as potential mechanical reinforcement agents in two hosts, polyvinyl alcohol (PVA) and poly(9-vinyl carbazole) (PVK). It was found that, by adding various concentrations of nanotubes, both Young's modulus and hardness increased by factors of 1.8 and 1.6 at $1 \mathrm{wt} \%$ in PVA and 2.8 and 2.0 at $8 \mathrm{wt} \%$ in PVK, in reasonable agreement with the Halpin-Tsai theory. Furthermore, the presence of the nanotubes was found to nucleate crystallization of the PVA. This crystal growth is thought to enhance matrix-nanotube stress transfer. In addition, microscopy studies suggest extremely strong interfacial bonding in the PVA-based composite. This is manifested by the fracture of the polymer rather that the polymer-nanotube interface. () 2002 American Institute of Physics. [DOI: 10.1063/1.1533118]
\end{abstract}

In recent years polymer-nanotube composites have been increasingly studied due to their interesting properties and potential for a wide range of applications. ${ }^{1-5}$ In these materials nanotubes (CNTs) are used as inclusions in various matrices to improve electrical, ${ }^{6,7}$ mechanical, ${ }^{8}$ or thermal properties. $^{9,10}$ In particular, due to their high Young's modulus ${ }^{11-13}$ CNTs are considered promising candidates for mechanical reinforcement of polymers. There are, however, a number of issues to be resolved, with the control of polymernanotube interfacial bonding as the main aim. Good interfacial bonding is essential to ensure efficient stress transfer from polymer matrix to nanotube lattice. Research into polymer-nanotube interfacial bonding has therefore been the topic of several studies. ${ }^{9,14-20}$

In this study, the increase in Youngs modulus $(Y)$ and hardness $(H)$ as a function of nanotube content are investigated by nanoindentation for two different polymer-CNT composites. Furthermore, the nature of the increase in mechanical properties is studied using differential scanning calorimetry (DSC) and transmission electron microscopy (TEM). It is found that the local polymer morphology and, hence, the macroscopic mechanical properties are strongly effected by the presence of nanotube inclusions.

The polymers used in this study were polyvinyl alcohol (PVA) and poly(9-vinyl carbazole) (PVK). These materials were purchased from Aldrich and used as supplied. A range of composite solutions were prepared by adding arc discharge multiwalled carbon nanotube (MWNT) soot (produced in TCD) to $30 \mathrm{~g} / 1$ solutions of PVA and PVK in their respective solvents, de-ionized water and dichloromethane. These solutions were mixed and purified as described

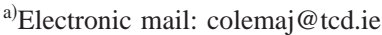

previously. ${ }^{21,22}$ True nanotube mass fractions were measured by thermogravimetric analysis (Mettler TA 4000) and were found to range between 0.1 and $1 \mathrm{wt} \%$ for PVA and 1.5 and $8 \mathrm{wt} \%$ for PVK. Films were formed on glass substrates by drop casting for PVA composites and spinning multiple layers in the case of PVK. Film thicknesses were measured by white light interferometry and were greater than one micron in all cases. Both DSC (TA Instruments DSC-2920) and thermogravimetric analysis measurements were carried out on free standing films with special care being taken to remove environmental moisture. In both cases the scan rate was 10 $\mathrm{K} / \mathrm{min}$. To perform transmission electron microscopy (Hitachi H-7000) formvar coated copper grids were dipped into composite solutions and allowed to dry in ambient conditions.

Mechanical properties were studied using nanoindention $^{23-25}$ (CSEM Nano Hardness Tester). Ten indentations were made and averaged for each sample. The maximum indentation force was $0.5 \mathrm{mN}$ with a loading/ unloading rate of $1 \mathrm{mN} / \mathrm{min}$ and indentation depth $<200 \mathrm{~nm}$ in all cases. As the nanotubes are distributed randomly in the film $Y$ can be modeled using the modified Halpin-Tsai equation [Eq. (1)] for randomly oriented fillers ${ }^{20,25}$

$$
\begin{aligned}
& Y_{C}=\left[\frac{3}{8} \frac{1+2\left(\frac{l_{N T}}{d_{N T}}\right) \eta_{L} V_{N T}}{1-\left(\eta_{L} V_{N T}\right)}+\frac{5}{8} \frac{1+\left(2 \eta_{T} V_{N T}\right)}{1-\left(\eta_{T} V_{N T}\right)}\right] Y_{M}, \\
& \eta_{L}=\frac{\left(\frac{Y_{N T}}{Y_{M}}\right)-1}{\left(\frac{Y_{N T}}{Y_{M}}\right)+2\left(\frac{l_{N T}}{d_{N T}}\right)}, \eta_{T}=\frac{\left(\frac{Y_{N T}}{Y_{M}}\right)-1}{\left(\frac{Y_{N T}}{Y_{M}}\right)+2},
\end{aligned}
$$



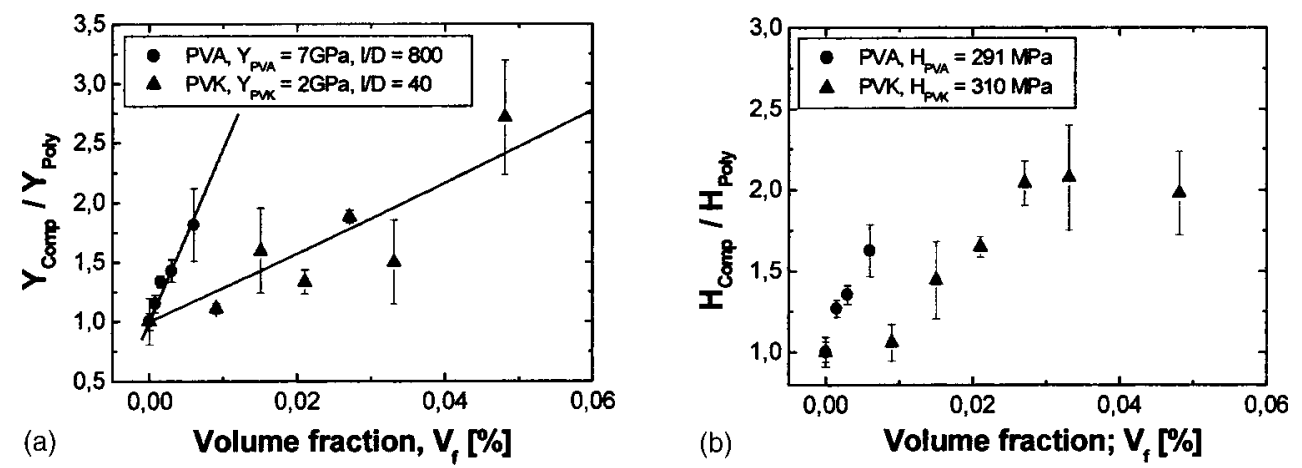

FIG. 1. (a) Young's modulus and (b) hardness for polyvinyl alcohol and poly(9-vinyl carbazole) composites.

where $(l / D)$ is the aspect ratio for the nanotubes and $V_{f}$ is the volume fraction of dispersed nanotubes. In order to fit these equations to the experimental data, mass fractions were transformed to volume fractions by assuming a density for perfectly graphitized MWNTs of $2.16 \mathrm{~g} / \mathrm{cm}^{3}$ (Ref. 20) and a polymer density of $1.3 \mathrm{~g} / \mathrm{cm}^{3}$ for PVA and PVK. In addition $Y$ for MWNTs was taken to be $1 \mathrm{TPa}$ in agreement with recent research studies. ${ }^{11-13}$ For the polymers $Y$ was found to be 7 and 2 GPa for PVA and PVK, respectively. Shown in Fig. 1(a) are normalized $Y$ values for both PVA and PVK plotted versus volume fraction of MWNTs in addition to fits to the Halpin-Tsai equation. It can clearly be seen that the values measured with nanoindentation can indeed be fit reasonably well with the earlier equation.

Significant increases in both $Y$ [Fig. 1(a)] and $H$ [Fig. 1(b)] were observed for both composite types. In case of the PVA based composite $Y$ increased from 7 to $12.5 \mathrm{GPa}$ as the nanotube content was increased to a loading level of $1 \mathrm{wt} \%$ $(0.6 \mathrm{vol} \%)$. For the same range of samples the hardness increased from 300 to $470 \mathrm{MPa}$. Similar results were observed for the PVK based composites, with $Y$ increasing from $2 \mathrm{GPa}$ for the pure PVK to $6 \mathrm{GPa}$ for the $8 \mathrm{wt} \%$ sample. Similarly $H$ values increased linearly from $300 \mathrm{MPa}$ for pure PVK to $600 \mathrm{MPa}$ for the $8 \mathrm{wt} \%(4.8 \mathrm{vol} \%)$ sample before leveling off.

As $Y_{N T}$ was assumed to be $1 \mathrm{TPa}$, the aspect ratio is a free parameter and can be obtained from a fit to the HalpinTsai equation. The $(l / D)$ values obtained were 800 and 40 for the PVA and PVK composites, respectively. This result is unexpected though as the same batch of MWNTs was used to produce each composite and the true aspect ratio, as measured by TEM is close to 100 . However, as the Halpin-Tsai equation is derived assuming perfect stress transfer it is likely that the difference in predicted aspect ratios is a mea- sure of the stress transfer for both matrices. This result shows that the interfacial bonding is far superior for the PVA matrix.

In order to understand the differences in interfacial bonding for the two host polymers DSC measurements were carried out. Shown in Fig. 2 are DSC traces for the PVA and PVK based composites, respectively. For PVA based composites, melting behavior is observed around $170{ }^{\circ} \mathrm{C}$. Analysis of this peak gives the percentage crystallinity of $14 \%$ for pure PVA. On the introduction of nanotubes, however, the crystallinity increases sharply to $24 \%$ before rising to $27 \%$ as the MWNT content is increased to $1 \mathrm{wt} \%$. This shows that the nanotubes act as nucleation sites for the crystallization of PVA in good agreement with previous conjugated polymerMWNT studies. ${ }^{26,27}$ In addition the presence of a shell of crystalline polymer around each nanotube appears to strongly enhance the stress transfer and, hence, the overall composite mechanical properties. Furthermore, the presence of this crystalline coating explains the unexpectedly large value for the aspect ratio suggested by Eq. (1) for the PVA based system. It is likely that the amplified crystallinity acts as an additional component of reinforcement, thus increasing the modulus above the levels expected from the nanotubes alone.

In contrast PVK composites show typical thermal behavior for amorphous materials. They exhibit only glass transition points with no melting peaks and, hence, no crystallinity is observed. While a second $T_{g}$ is observed for the PVK composites this suggests the creation of a second phase but not a crystalline one. These results, in conjunction with the mechanical measurements strongly suggests that the interaction of an amorphous polymer with nanotubes results in poor stress transfer. This is despite the fact that the conjugated
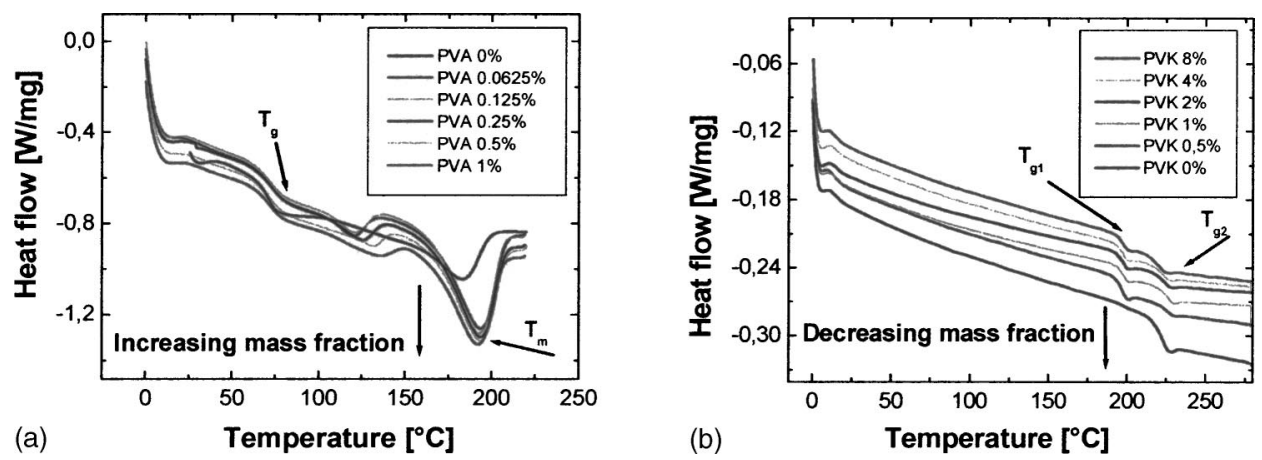

FIG. 2. Differential scanning calorimetry of (a) polyvinyl alcohol and (b) poly(9-vinyl carbazole) composites.

Downloaded 01 Jul 2010 to 134.226.1.229. Redistribution subject to AIP license or copyright; see http://apl.aip.org/apl/copyright.jsp 


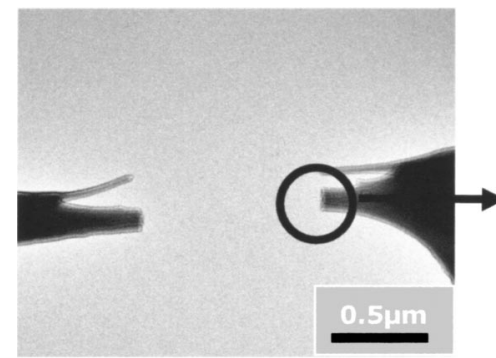

(a)

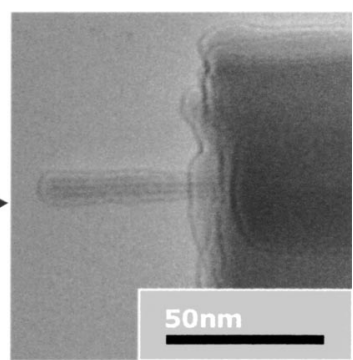

(b)
FIG. 3. Transmission electron micrographs of MWNT pullout from PVA.

nature of PVKs sidechains would suggest strong interaction with nanotubes.

In order to understand the fracture mechanism in the MWNT/PVA composite TEM studies were performed. Shown in Fig. 3(a) is a TEM micrograph of a PVA film after tearing due to exposure to a concentrated electron beam in the microscope. On magnification of the right side of Fig. 3(a) a nanotube can be observed protruding from the polymer fracture zone. It is likely that local heating of the film by the beam caused contraction resulting in the nanotube being pulled from the polymer. However, a higher magnification image, shown in Fig. 3(b) clearly shows this nanotube coated with a thin layer of crystalline polymer. This is a remarkable result as it shows that the polymer did not break at the polymer-nanotube interface suggesting extremely strong interfacial interaction.

In summary, mechanical and thermal properties of amorphous and semicrystalline MWNT reinforced polymer films have been studied by nanoindention and DSC. In both cases significant increases in $Y$ and $H$ were observed. In terms of morphology, the crystallinity of PVA increased significantly on addition of $1 \mathrm{wt} \%$ (0.6 vol \%) MWNTs. In contrast no crystallization was observed in PVK. The nucleation of crystal PVA results in an extremely strong interfacial interaction between nanotube and polymer and is the major reason for the reinforcement of PVA. Furthermore, TEM micrographs clearly show that when under stress, the polymer matrix failed rather than the polymer nanotube interface. This indicates an excellent interfacial interaction, suggesting that nanotubes are exceptional candidates for reinforcement of PVA.

The support of the European Union NANOCOMP Network Program under Contract No. HPRN-CT-2000-00037 is gratefully acknowledged. Furthermore, Professor V. Altstädt is kindly acknowledged for providing equipment for DSC.

${ }^{1}$ S. A. Curran, P. M. Ajayan, W. J. Blau, D. L. Carroll, J. N. Coleman, A. B. Dalton, A. P. Davey, A. Drury, B. McCarthy, S. Maier, and A. Strevens, Adv. Mater. 10, 1091 (1998).

${ }^{2}$ M. S. P. Shaffer and A. H. Windle, Adv. Mater. 11, 937 (1999).

${ }^{3}$ B. McCarthy, J. N. Coleman, R. Czerw, A. B. Dalton, M. i. h. Panhuis, A. Maiti, A. Drury, P. Bernier, J. Nagy, B. Lahr, H. J. Byrne, D. L. Carroll, and W. J. Blau, J. Phys. Chem. B 106, 3087 (2002).

${ }^{4}$ M. Cadek, B. le Foulgoc, J. N. Coleman, V. Barron, J. Sandler, M. S. P. Shaffer, A. Fonseca, M. van Es, K. Schulte, and W. J. Blau, AIP Conf. Proc. 633, 562 (2002).

${ }^{5}$ B. Vigolo, P. Poulin, M. Lucas, P. Launois, and P. Bernier, Appl. Phys. Lett. 81, 1210 (2002).

${ }^{6}$ B. E. Kilbride, J. N. Coleman, J. Fraysse, P. Fournet, M. Cadek, A. Drury, S. Hutzler, S. Roth, and W. J. Blau, J. Appl. Phys. 92, 4024 (2001).

${ }^{7}$ J. Sandler, M. S. P. Shaffer, T. Prasse, W. Bauhofer, K. Schulte, and A. H. Windle, Polymer 40, 5967 (1999).

${ }^{8}$ R. Haggenmueller, H. H. Gommans, A. G. Rinzler, J. E. Fischer, and K. I. Winey, Chem. Phys. Lett. 330, 219 (2000).

${ }^{9}$ M. J. Biercuk, M. C. Llaguno, M. Radosavljevic, J. K. Hyun, J. E. Fischer, and A. T. Johnson, Appl. Phys. Lett. 80, 2767 (2002).

${ }^{10}$ C. Wei, D. Srivastava, and K. Cho, Nano Lett. 2, 647 (2002).

${ }^{11}$ M. M. J. Treacy, T. W. Ebbesen, and J. M. Gibson, Nature (London) 381, 678 (1996).

${ }^{12}$ E. W. Wong, P. E. Sheehan, and C. M. Lieber, Science 277, 1971 (277).

${ }^{13}$ O. Lourie and H. D. Wagner, Appl. Phys. Lett. 73, 3527 (1998).

${ }^{14}$ P. M. Ajayan, L. S. Schadler, C. Giannaris, and A. Rubio, Adv. Mater. 12, 750 (2000).

${ }^{15}$ P. M. Ajayan, O. Stephan, C. Colliex, and D. Trauth, Science 265, 1212 (1994).

${ }^{16}$ O. Lourie and H. D. Wagner, Appl. Phys. Lett. 73, 3527 (1998).

${ }^{17}$ O. Lourie, D. M. Cox, and H. D. Wagner, Phys. Rev. Lett. 81, 1638 (1998).

${ }^{18}$ H. D. Wagner, O. Lourie, Y. Feldman, and R. Tenne, Appl. Phys. Lett. 72, 188 (1998).

${ }^{19}$ C. Bower, R. Rosen, L. Jin, J. Han, and O. Zhou, Appl. Phys. Lett. 74, 3317 (1999).

${ }^{20}$ D. Qian, E. C. Dickey, R. Andrews, and T. Rantell, Appl. Phys. Lett. 76, 2868 (2000).

${ }^{21}$ J. N. Coleman, D. F. O'Brien, A. B. Dalton, B. McCarthy, B. Lahr, A. Drury, and W. J. Blau, Chem. Commun. (Cambridge) 20, 2001 (2000).

${ }^{22}$ R. Murphy, J. N. Coleman, M. Cadek, B. McCarthy, M. Bent, A. Drury, R. C. Barklie, and W. J. Blau, J. Phys. Chem. B 106, 3087 (2001).

${ }^{23}$ W. Herrera-Kao and M. Aguilar-Vega, Polym. Bull. (Berlin) 42, 449 (1999).

${ }^{24}$ W. C. Oliver and G. M. Pharr, J. Mater. Res. 7, 1564 (1992).

${ }^{25}$ P. K. Mallick, Fibre-Reinforced Composites (Marcel Dekker, New York, 1993), p. 130.

${ }^{26}$ J. N. Coleman, K. P. Ryan, M. S. Lipson, A. Drury, M. Cadek, M. i. h. Panhuis, R. P. Wool, and W. J. Blau, AIP Conf. Proc. 633, 557 (2002).

${ }^{27}$ K. P. Ryan, S. M. Lipson, S. M. O'Flaherty, V. Barron, M. Cadek, A. Drury, H. J. Byrne, R. P. Wool, W. J. Blau, and J. N. Coleman, SPIE OptoIreland, Galway, Ireland, 2002, Vol. 4876. 